\section{Síndrome de Goldenhar y microsomía hemifacial. Revisión de la literatura}

\section{Goldenhar syndrome and hemifacial microsomia. A literature review}

\begin{abstract}
Resumen
La microsomía hemifacial corresponde a la segunda malformación congénita en prevalencia, luego de la fisura labiopalatina, y se describe como una alteración congénita del primer y el segundo arcos branquiales. El síndrome de Goldenhar tiene características típicas de la microsomía hemifacial, con la adición de dermoides epibulbares y anomalías vertebrales. Ambas patologías presentan características de expresión variable y por tanto los tratamientos son acordes a su individualidad. En esta revisión de tema se analizan su etiología, clasificaciones, características y tratamiento.
\end{abstract}

Palabras clave: Síndrome de Goldenhar; Anomalías maxilofaciales; Anomalías maxilomandibulares.

\begin{abstract}
Hemifacial microsomia is the second congenital malformation in prevalence, after cleft lip and palate, and is described as a congenital alteration of the first and second branchial arches. Goldenhar syndrome has typical characteristics of hemifacial microsomia with the addition of epibulbar dermoids and vertebral anomalies. Both pathologies have variable expression's characteristics and therefore the treatments are according to their individuality. In this review of the topic is analyzed the etiology, classifications, characteristics and treatment.
\end{abstract}

Keywords: Goldenhar syndrome; Maxillofacial abnormalities; Jaw abnormalities.
ISSN: $1560-9111$

\section{Artículo de Revisión}

\author{
Lissette Cazenave ${ }^{1, a}$, Noemi Leiva ${ }^{2, b}$, Carmen \\ Gloria Morovic ${ }^{3,0}$ \\ ${ }^{1}$ Facultad de Odontología. Universidad de Chi- \\ le. Santiago, Chile. \\ ${ }^{2}$ Hospital Luis Calvo Mackenna. Santiago, Chile. \\ ${ }^{3}$ Facultad de Medicina. Universidad de Chile. \\ Santiago, Chile. \\ ${ }^{a}$ Cirujano Dentista. \\ b Ortodoncista \\ ${ }^{\mathrm{C}}$ Cirujano Plástico Pediátrico
}

Correspondencia:

Lissette Cazenave Lafertte.

Correo electrónico: lissettecazenave@gmail.com General Holley 2381 of 606 B Providencia.

Santiago, Chile.

\section{Coautores:}

Noemi Leiva

leivanoemi@yahoo.com

Carmen Gloria Morovic

cmorovic@gmail.com

Conflicto de intereses: Los autores declaran no tener conflictos de interés.

Fuente de financiamiento: Autofinanciado.

Fecha de recepción: 16/08/17

Fecha de aceptación: 09/09/17

\section{Introducción}

La microsomía hemifacial (MHF) incluye un espectro de malformaciones que involucran principalmente estructuras derivadas del primer y segundo arcos branquiales. Los hallazgos característicos incluyen asimetría facial leve, moderada y severa resultado de una hipoplasia unilateral maxilar y/o mandibular; apéndices preauriculares o faciales; malformaciones de pabellón auricular que pueden incluir microtia (hipoplasia del oído externo), anotia (ausencia del oído externo), o atresia aural (ausencia de conducto auditivo externo); y pérdida de audición y malformaciones oculares ${ }^{1}$, pero su característica clínica principal es la asimetría facial, particularmente de la mandíbula. Ocurre bilateralmen- te en el 10\%-16\% de los casos, en los cuales se prefiere el término microsomía cráneo facial (MCF), y afecta más a hombres que a mujeres, en una relación de 3:2, y más al lado derecho que el izquierdo, en proporción de $3: 2^{2}$. Las personas con MHF y dermoides epibulbares se dice que tienen síndrome de Goldenhar (SG) o displasia oculoauriculo vertebral. La triada patognomónica del SG incluye MHF, dermo-lipoma y anormalidades esqueletales vertebrales ${ }^{1,3}$.

Un análisis de las diferencias objetivas entre pacientes diagnosticados con SG y con MHF, reveló que existen más pacientes diagnosticados con SG que aquellos con quistes dermoides epibulbares y anomalías vertebrales. Además, no todos los pacientes que presentaban alguna de estas características fueron subjetivamente diag- 
nosticados con SG. También, los pacientes considerados subjetivamente con diagnóstico de SG tenían una mandíbula más severamente afectada, deformación del tejido blando y eran más propensos a tener compromiso bilateral y macrostomia. Sin embargo, al examinar más apropiadamente las características objetivas del SG, el grado de severidad de casi todas las deformaciones cráneo faciales de estos pacientes no eran significativamente diferentes ${ }^{3}$.

\section{Etiología}

La causa de la MHF permanece desconocida, pero parece ser un defecto en el desarrollo del primer y segundo arco faríngeo durante las primeras 6 semanas de gestación. Poswillo obtuvo el fenotipo de la MCF en ratones, administrando teratógenos que causaban un hematoma en la arteria estapedia y la arteria del segundo arco, lo que resultó en una necrosis regional. El amplio espectro de anomalías faciales parece ser producido, por lo tanto, por la extensión de la lesión tisular debido a la necrosis y su habilidad de regenerar. Estudios más recientes han demostrado una asociación entre la ocurrencia de MCF y la gestación múltiple, además de los siguientes factores de riesgo de la madre: uso de medicamentos vasoactivos, tabaquismo durante el segundo trimestre de embarazo, diabetes mellitus y utilización de tecnología reproductiva asistida. Además, se han descrito patrones de transmisión autosómico dominante y recesivo en familias con características de MHF, y se ha observado una historia familiar positiva del $50 \%$ en muchas series de casos. También se ha descrito una variedad de anormalidades genéticas. Es posible que la heterogeneidad etiológica junto con la variabilidad en la penetrancia genética y expresión puedan contribuir al amplio espectro fenotípico visto en la MCF ${ }^{2,4,5}$.

La etiopatogenia del SG permanece aún poco conocida y en muchos casos sin explicación. Se sospecha que la razón de ocurrencia de este síndrome es multifactorial y depende de factores genéticos y ambientales. En la literatura se puede encontrar información acerca de casos con herencia autosómica dominante o recesiva. Algunos autores estiman que la ocurrencia de SG en pacientes con historia familiar positiva del síndrome es de un mínimo porcentaje pero otros identifican incluso un 31\% de casos familiares. Algunos estudios también muestran que los parientes de primer grado (entre hermanos o desde los padres a los hijos) fueron mayormente afectados. El riesgo de recurrencia de este síndrome probablemente alcanza el 2\%-3\%. Sin embargo, la mayoría de los casos descritos del síndrome aparecen esporádicamente. Algunos investigadores sugieren que el origen de este síndrome se debe a un desarrollo anormal de la vascularización en la cuarta semana de embarazo, cuando sucede el desarrollo del primer y segundo arco faríngeo, responsables del crecimiento de las estructuras craneofaciales. Además, muchos factores externos como medicamentos vasoactivos, tabaquismo, consumo de cocaína, exposición a talidomida, terapia hormonal, drogas para el tratamiento de algunas enfermedades como el medicamento antineoplásico tamoxifeno podrían contribuir en la interferencia del crecimiento normal del primer y segundo arco faríngeo. Algunos estudios han demostrado que los hijos de madres diabéticas son más propensos a desarrollar el síndrome. El riesgo aumentado de desarrollar el síndrome además se relaciona firmemente con el hipotiroidismo maternal, enfermedad celíaca, sangrado vaginal durante el embarazo o parto prematuro. Uno de los artículos ha demostrado una falta de asociación entre la edad de los padres y la ocurrencia de SG, duración del ciclo menstrual o casos previos de aborto espontáneo. Sin embargo, existe una correlación estadísticamente significativa entre el embarazo a una edad más avanzada de ambos padres y una mayor frecuencia de nacimientos de nińos con SG. Se ha observado un riesgo aumentado en el caso de múltiples embarazos, especialmente con gemelos. Además, los defectos estructurales afectaron dos o tres veces más frecuentemente a los gemelos monocigóticos. Algunos investigadores señalan una mayor recurrencia de SG en embarazadas por fertilización in vitro $^{2,6,7}$.

\section{Clínica - Características y presentación}

No existe un criterio establecido para el diagnóstico de MHF, ya que se presenta con un amplio fenotipo, pudiendo quedar sin diagnosticar los casos más leves. Típicamente se presenta con asimetría mandibular (unilateral o bilateral) (Figura 1), distopia orbitaria (asimetría vertical de los ojos), macrostomía (amplia apertura bucal) y microtia (orejas pequeñas) ${ }^{2}$. Muchos estudios han indicado que los defectos mandibulares o auriculares son mandatorios para el diagnóstico. La deficien-
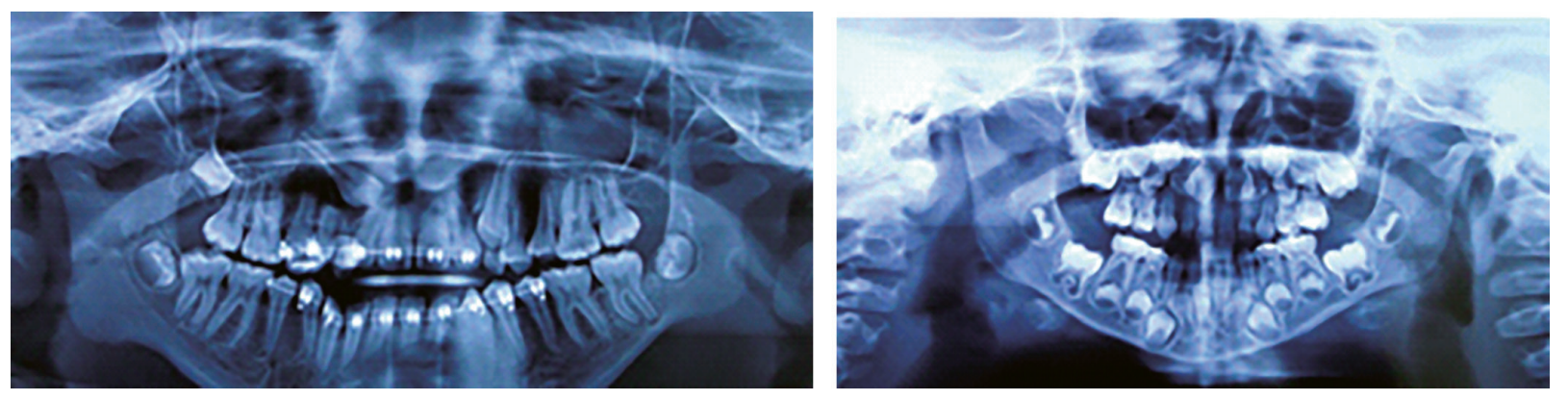

Figura 1. Ortopantomografías de pacientes con microsomía hemifacial. 
cia mandibular puede ir desde la ausencia del cartílago condilar y disco a una completa falla del desarrollo de la rama. El maxilar, el hueso temporal y la órbita también pueden estar afectados como resultado de malformación primaria. Sin embargo, la MHF no se caracteriza por dimorfismo óseo por sí solo, ya que también hay compromiso de tejido blando neural y muscular ${ }^{8,9}$. Otras características menos frecuentes incluyen pequeños apéndices de piel anteriores al tragus de la oreja, fisura labiopalatina en el 10\%-25\% de los casos, talla pequeña para la edad, parálisis del nervio facial en el 25\%-40\% de los casos, problemas de la vía aérea como SAHOS, anoftalmia, desarrollo dental tardío e incidencia aumentada de microdoncia ${ }^{2}$.

Según Pruzansky (1969), las deformidades mandibulares en la MHF se clasifican en:

- Grado I: presenta una hipoplasia leve mandibular, todas las estructuras se encuentran presentes.

- Grado II: el cóndilo y la rama son más pequeños, la cabeza del cóndilo es plana, con ausencia de cavidad glenoídea; la apófisis coronoide puede estar ausente.

- Grado III: la rama mandibular puede estar reducida a una pequeña y delgada lámina de hueso o no existir.

La clasificación de Pruzansky fue posteriormente modificada por Kaban (1988), agregando subcategorías al grado II, quedando de la siguiente manera:

- Tipo I: la morfología es normal, pero la cavidad glenoidea, rama ascendente y cóndilo son de menor tamaño (mandíbula pequeña).

- Tipo II: la ATM, rama ascendente y fosa glenoidea son hipoplásicos y existe malformación de las mismas. La fosa glenoidea puede estar malposicionada.

- II a: relación cóndilo fosa es mantenida, ATM funcional, condición aceptable para la característica asimétrica.

- II b: relación cóndilo fosa no mantenida, malposición en los tres planos del espacio, ATM no funcional, muy hipoplásica y desplazada en sentido mesial anterior e inferior en relación con el otro lado.

Tipo III: ausencia tanto de la rama ascendente como de la fosa glenoidea y del cóndilo (ATM ausente) ${ }^{10-12}$.

La clasificación de Pruzansky ha sido por décadas el sistema más ampliamente utilizado para clasificar las deformidades mandibulares de los pacientes con MHF, pero al cumplir sólo con la descripción de la mandíbula, quedan fuera muchos aspectos propios de la patología ${ }^{11}$.

Vento (1991) creó una clasificación que incluye tejido duro, blando y función - OMENS (órbita (Orbit), mandíbula (Mandible), oído (Ear), nervio (Nerve) y deficiencias del tejido blando (Soft tissue)). A cada categoría se le asigna un puntaje (0-3), siendo 3 el puntaje para la deformación más severa, obteniendo un puntaje total de deformación, donde 15 es el máximo posible ${ }^{2}$.

\section{Clasificación OMENS ${ }^{11-13}$}

O: Asimetría de la órbita (Orbit)

OO: Órbita con tamaño y posición normales.

O1: Tamaño orbitario anormal.

O2: Posición orbitaria anormal (colocar una flecha de la posición, ej: $\mathrm{O} 2 \uparrow$ si es superior, $\mathrm{O} 2 \downarrow$ si es inferior).

O3: Posición y tamaño orbitario anormales.

\section{M: Hipoplasia mandibular (Mandible)}

M0: Mandíbula normal.

M1: La mandíbula y la fosa glenoídea son pequeñas, con una rama corta.

M2A: La fosa glenoídea tiene una posición anatómicamente aceptable con referencia a la ATM opuesta.

M2B: La ATM está desplazada inferior, medial y anteriormente, con un cóndilo severamente hipoplásico.

M3: Existe ausencia completa de rama, fosa glenoídea y ATM.

\section{E: Deformidad en el oído externo (Ear)}

E0: Oreja normal.

E1: Hipoplasia leve, pero todas las estructuras están presentes.

E2: Ausencia de canal auditivo externo con hipoplasia variable de la concha.

E3: Lóbulo mal posicionado, con ausencia de oreja. El remanente lobular generalmente está desplazado inferior $y$ anteriormente.

\section{$\mathrm{N}$ : Compromiso de los nervios (Nerve)}

N0: No existe compromiso del nervio facial.

N1: Compromiso superior del nervio facial (ramas temporal y cigomática).

N2: Compromiso inferior del nervio facial (ramas bucal, mandibular y cervical).

N3: Todas las ramas del nervio facial están afectadas. Se pueden analizar otros nervios comprometidos, como el trigémino $\mathrm{V}$ par (sensorial), el hiplogloso XII par, y al resto de los nervios craneales se les anota con su propio número.

\section{S: Deficiencia en tejido blando (Soft tissue)}

S0: No existe deficiencia en tejido blando ni muscular.

S1: Deficiencia mínima de tejidos blandos y muscular.

S2: Deficiencia moderada, entre los dos extremos, S1 y S3. 
S3: Severa deficiencia de tejidos blandos debida a hipoplasia de tejido celular subcutáneo y del músculo.

La clasificación OMENS ha sido sometida a modificaciones posteriores, postulándose la OMENS+ (1995), con el fin de ampliar la expresión fenotípica de los pacientes con MHF hacia las alteraciones extracraneales. Y se postuló además una representación pictográfica de la OMENS + (2007), la cual fue luego modificada en 2011, con el fin de facilitar su comprensión en la práctica clínica, en la docencia y en la estandarización de la clasificación de pacientes con MHF ${ }^{11-13}$.

En cuanto a SG, se dice que representan el $10 \%$ de los casos de MHF, y que consiste en la triada de:

- MHF.

- Anomalías vertebrales.

- Anomalías oculares - dermoides epibulbares y/o coloboma del párpado superior.

Es típicamente una condición unilateral, a diferencia de la $\mathrm{MCF}^{2}$ (Figuras 2 y 3 ).
Las características clásicas del síndrome incluyen alteraciones oculares como microftalmia, dermoides epibulbares, lipodermoides y coloboma; alteraciones auriculares como apéndices pre-auriculares, pérdida auditiva y microtia; y anomalías vertebrales como escoliosis, hemivértebras y fusión cervical. Las anomalías han sido reportadas unilaterales en el $85 \%$ de los casos y bilaterales en el $10-33 \%$ de los casos. Las anomalías oculares, especialmente los dermoides bilaterales han sido observados en el $60 \%$ de los casos, las anomalías vertebrales en el $40 \%$ de los casos y las anomalías auriculares en el $40 \%$ o más de los casos. Se han observado otras características sistémicas en el $50 \%$ de los pacientes. La tetralogía de Fallot y los defectos de tabique ventricular son las anomalías cardiovasculares más frecuentes asociadas a SG. El labio y paladar con fisura, macrostomia, micrognatia, cuello corto, fístulas traqueoesofágicas, anomalías del músculo esternocleidomastoideo, hipoplasia pulmonar, hernia umbilical, hernia inguinal, anomalías renales, vagina hipoplásica y anomalías anales podrían estar asociadas al síndrome. La anoftalmia, parálisis facial, calcificación del falx cerebri, criptorquídea y asociación del SG

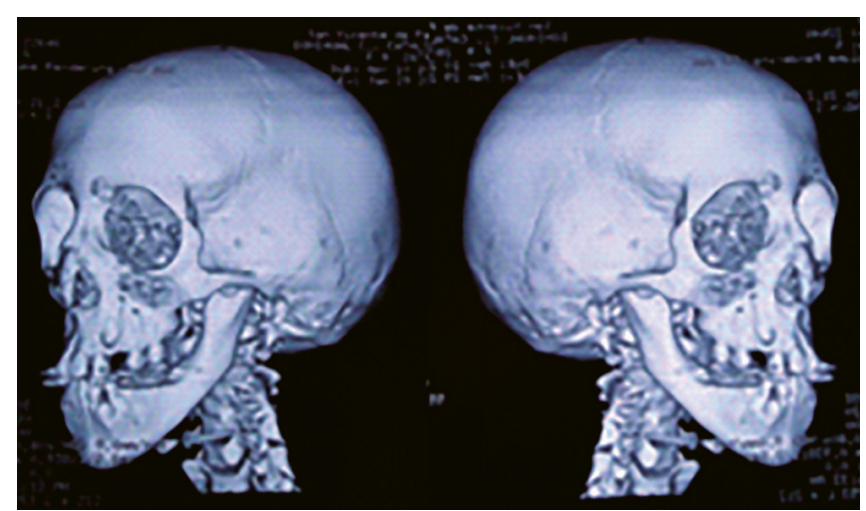

Figura 2. TAC de cráneo, reconstrucción 3D, paciente con síndrome de Goldenhar. Nótese la agenesia de ambos conductos auditivos externos.

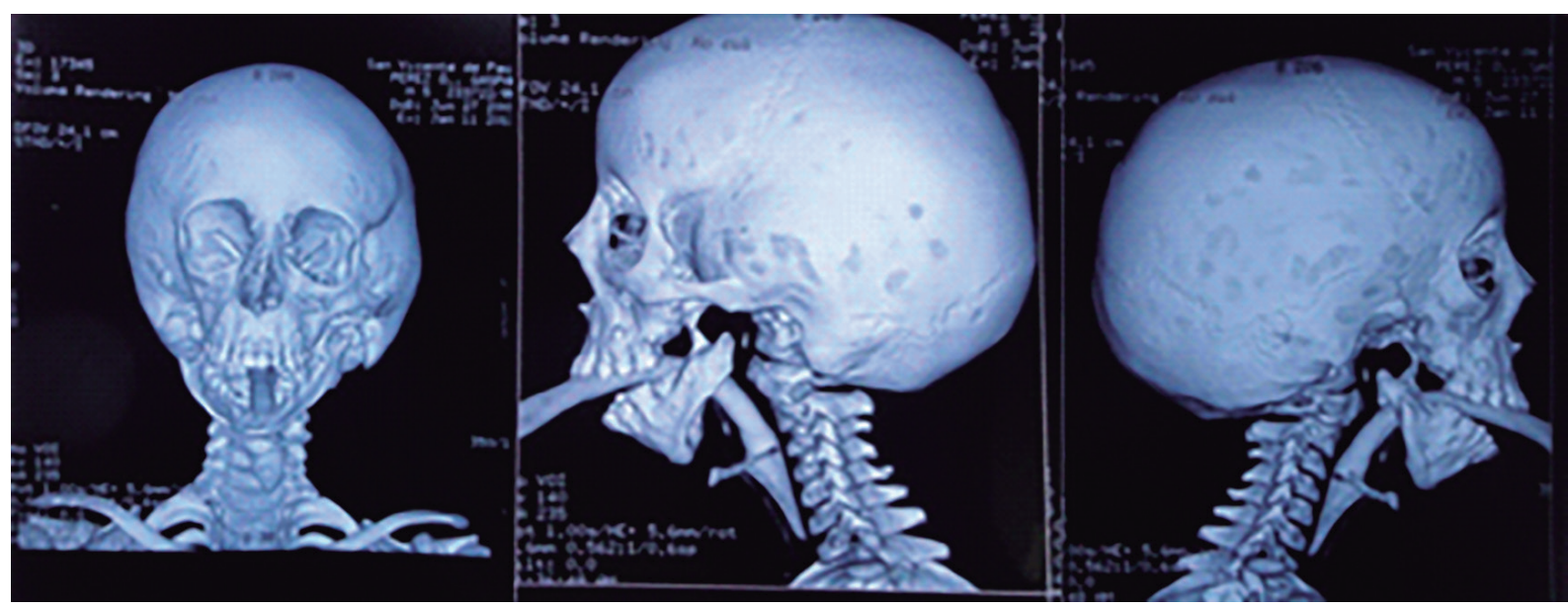

Figura 3. TAC de cráneo, reconstrucción 3D, paciente con síndrome de Goldenhar. Nótese la hipoplasia del maxilar, macroglosia, hipertrofia de la úvula con obstrucción de la vía aérea a nivel orofaríngeo posterior, agenesia de conducto auditivo externo y pabellón auricular derecho. 
Tabla 1. Presentación clínica de síndrome de Goldenhar y microsomía hemifacial ${ }^{5,6,11}$

\begin{tabular}{ll}
\hline \multicolumn{2}{c}{ Síndrome de Goldenhar } \\
\hline Dermoidesepibulbares & Manifestaciones oculares \\
Párpado fisurado & Dermoidesepibulbares \\
Microftalmia & Blefaroptosis \\
Exoftalmia & Microftalmia \\
Anoftalmia & Distopía orbitaria (mala posición) \\
Estrabismo & Coloboma retinal o coroidal \\
Asimetría/dismorfia ocular & \\
Coloboma & \\
Lipodermoides & \\
Atresia/estenosis del conducto lacrimal & \\
Dacriocistitis &
\end{tabular}

Dacriocistitis

\begin{tabular}{ll}
\hline & Manifestaciones auriculares \\
\hline Anotia & Anotia \\
Microtia & Microtia \\
Asimetría auricular & Pérdida de audición \\
Atresia del canal auditivo externo & Hipoacusia \\
Apéndices preauriculares & Alteraciones del oído medio \\
Displasia auricular con o sin pérdida auditiva & \\
Fístulas preauriculares & \\
Alteraciones de oído interno y medio & \\
\hline
\end{tabular}

Alteraciones de oído interno y medio

\begin{tabular}{|c|c|}
\hline \multicolumn{2}{|c|}{ Deformaciones craneofaciales } \\
\hline Deformaciones craneofaciales & Fisura labio palatina \\
\hline Alteraciones del primer y segundo arco faríngeo & Macrostomía \\
\hline Asimetría facial & Hipoplasia de los tercios faciales \\
\hline Fisura facial & Inclinación del plano oclusal ( canteado ) \\
\hline Hipoplasia mandibular y/o maxilar & Hipoplasia de los músculos masticatorios \\
\hline Microsomíahemifacial & Insuficiencia velofaríngea \\
\hline Labio con fisura & Agenesias dentales \\
\hline Paladar con fisura & Hipoplasias dentales \\
\hline Macrostomía & Microdoncia \\
\hline Lengua bífida & Maloclusiones \\
\hline Maloclusiones & Retraso en el desarrollo dental en pacientes con MHF tipo IIB y III \\
\hline Discrepancias dentales & (afecta principalmente a los dientes posteriores) \\
\hline Agenesia de 2dos premolares y 3eros molares & \\
\hline Dientes supernumerarios & \\
\hline Malformaciones de esmalte y dentina & \\
\hline Retraso en el desarrollo dental & \\
\hline \multicolumn{2}{|c|}{ Alteraciones esqueletales } \\
\hline Espina bífida & Vértebras en mariposa \\
\hline Microcefalia & Hemivértebra \\
\hline Dolicocefalia & Fusión vertebral \\
\hline Plagiocefalia & Vertebra fisurada \\
\hline Defectos vertebrales & Espina bífida \\
\hline Pie equinovaro & Escoliosis \\
\hline Hemimelia radial & Anomalías de costillas \\
\hline \multirow[t]{12}{*}{ Alteraciones del dedo pulgar } & Defectos vertebrales \\
\hline & Sinus (quiste) pilonidal o decoloración de la piel paravertebral \\
\hline & Defectos estructurales de piernas y brazos \\
\hline & Hemimelia radial \\
\hline & Hipoplasia del pulgar \\
\hline & Pie equinovaro \\
\hline & Hipoplasia monolateral de la cresta iliaca \\
\hline & Desviación ulnar de la mano \\
\hline & Hombros asimétricos \\
\hline & Síndrome de Klippel-Feil \\
\hline & Dismetría de piernas \\
\hline & Clinodactilia/sindactilia \\
\hline
\end{tabular}




\begin{tabular}{ll}
\hline & Alteraciones en órganos internos \\
\hline Anomalías cardiovasculares: & Anomalías cardiovasculares: \\
Defectos del tabique auricular y ventricular (los más frecuentes) & Coartación aórtica \\
Defectos conotruncales & Dextrocardia \\
Tetralogía de Fallot & Enfermedades de las válvulas del corazón \\
Comunicación arterioso persistente & Alteraciones vasculares \\
Anomalías del arco aórtico & Defectos del tabique auricular y ventricular \\
Otras alteraciones en la vía de salida & Ducto arterioso persistente \\
Transposición de los grandes vasos & \\
Dextrocardia & \\
\end{tabular}

\begin{tabular}{ll}
\hline Alteraciones urogenitales: & Alteraciones urogenitales: \\
Riñones ectópicos & Riñones ectópicos \\
Riñones fusionados & Displasia poliquística renal \\
Uréter doble & Hipoplasia renal \\
Hidronefrosis & Riñones ectópicos \\
Agenesia renal & Agenesia renal \\
Riñones poliquísticos & Riñones en herradura (fusionados) \\
Hidroureter & Criptorquidia \\
& Reflujo vesicoureteral \\
& Anomalías menores (hernia inguinal, hidrocele, varicocele,
\end{tabular}

$$
\text { megaureter) }
$$

\section{Sistema Nervioso Central:}

Hipoplasia cerebral difusa

Hidrocefalia debido a estenosis del acueducto de Silvio

Ventrículos laterales cerebrales dilatados o hidrocefalia asintomática

Lipoma del cuerpo calloso

Ventrículos laterales asimétricos

Ausencia del septum pellucidum

Disgenesia del cuerpo calloso

Hipodensidad cerebral difusa

Hipodensidades frontales

Parálisis facial

Microcefalia

Anestesia trigeminal

Encefalocele

Deformidades de columna vertebral

Holoprosencefalia

Malformación de Arnold-Chiari

Hamartoma hipotalámico

Aplasia/hipoplasia de las articulaciones temporomandibulares

\begin{tabular}{ll}
\hline Tracto gastrointestinal: & Tracto gastrointestinal: \\
Atresia rectal & Atresia esofágica \\
Fístula traqueoesofágica & Atresia duodenal \\
Atresia esofágica & Estenosis pilórica \\
& Estenosis esofágica \\
& Malformaciones anales-rectales \\
& Hernia diafragmática \\
& Vólvulo intestinal congénito \\
& Anomalías menores (hernia umbilical y reflujo gastroesofágico)
\end{tabular}

\section{Sistema respiratorio:}

Anatomía anormal de laringe y faringe

Trastorno de la anatomía lobular de los pulmones.

\section{Sistema Nervioso Central:}

Agenesia o anormalidad del cuerpo calloso

Hidrocefalia

Síndrome de Arnold-Chiari

Quistes cerebrales
Anomalías menores (hernia umbilical y reflujo gastroesofágico)

\section{Sistema respiratorio:}

Aplasia/hipoplasia pulmonar

Laringomalacia congénita

Malformación funcional/estructural faríngea-laríngea

Defectos de asimetría en la nariz

Fístula de la piel nasal. 
con síndrome de Turner y glaucoma son asociaciones reportadas con menos frecuencia ${ }^{3,14}$.

En la tabla 1 se presenta un resumen comparativo de la presentación clínica de SG y MHF.

\section{Tratamiento - Antecedentes}

El manejo de la MHF es complejo y de larga duración. Problemas severos de la vía aérea pueden requerir intubación e intervención quirúrgica temprana en términos de distracción ósea mandibular para mejorar la posición mandibular o incluso traqueotomía ${ }^{2}$. Los pacientes más severamente afectados podrían mostrar signos de SAHOS ${ }^{15}$. La reparación de los tejidos blandos de los apéndices de piel y la macrostomía es usualmente llevada a cabo durante el primer ańo de vida. Los problemas de oído medio e interno son abordados según sea necesario para promover el correcto desarrollo del habla y lenguaje. La reconstrucción de la oreja normalmente se retrasa hasta que ha completado casi totalmente su crecimiento (7-8 años). Los dermoides epibulbares pueden ser resecados o reducidos pero no sin riesgo ${ }^{2}$.

Los casos más leves (Pruzansky I), podrían ser tratados en dentición mixta con aparatos de ortodoncia removibles o fijos, para maximizar el potencial de crecimiento del maxilar y mandíbula en el lado afectado. Existe cierto debate en cuanto al éxito del tratamiento interceptivo con aparatos funcionales dada las deficiencias de los tejidos blandos y duros subyacentes. Actualmente, muchos de los casos más leves no requieren intervención alguna en dentición mixta ni permanente temprana. Las deformaciones del tipo Pruzansky II han sido tratadas tradicionalmente con distracción osteogénica de la mandíbula descrita por McCarthy en la dentición mixta. El trabajo de Meazzini y otros ha mostrado sin embargo que los beneficios estéticos y psicológicos pueden ser de breve duración ya que la mandíbula tiende a volver a su posición original y la asimetría reaparece. Como la deformidad es tridimensional, es una condición difícil de manejar, especialmente en un niño en crecimiento. La práctica actual se aleja de la intervención en la dentición mixta ${ }^{2}$. Sin embargo, la distracción osteogénica mandibular ha demostrado ser exitosa en pacientes con dimorfismo leve a moderado siempre que exista un programa clínico integral que haga hincapié en mantener un stock de hueso mandibular adecuado en caso de ser necesarias futuras intervenciones, una selección apropiada de vectores, sobre-corrección y manejo integral de ortodoncia ${ }^{16}$.

Los individuos más severamente afectados (Pruzansky III), usualmente requieren intervención quirúrgica con un injerto costocondral (costilla o similar) para fomentar el crecimiento mandibular y maximizar el potencial de crecimiento en el maxilar. Esto a menudo se lleva a cabo a los 7-10 años de edad. Es sabido, sin embargo, que el comportamiento del crecimiento costocondral en la mandíbula es impredecible; un tercio de las costillas no crece, un tercio crece a un rango normal y el tercio restante presenta sobrecrecimiento ${ }^{2}$.
Un estudio retrospectivo evidenció que para la MHF en casos de pacientes jóvenes, lo más recomendable es esperar que se complete el crecimiento antes de realizar distracción osteogénica mandibular, ya que si bien la distracción osteogénica es efectiva para el alargamiento de la rama de la mandíbula hipoplásica, ofreciendo una clara mejoría en la apariencia facial, simetría y oclusión dental, y mejora además la estética y autoestima del paciente en el corto plazo, se ha observado a largo plazo una recidiva de la asimetría facial cuando se realiza a edades tempranas, requiriendo dichos pacientes de cirugía ortognática en un futuro para corregir la asimetría ${ }^{17}$.

Una revisión sistemática reciente de Pluijmers ha encontrado que no existe evidencia estadísticamente significativa que apoye el uso de distracción osteogénica como modalidad única de tratamiento en niños. Los mejores resultados se observaron en los casos más leves tratados con distracción osteogénica e injertos en la dentición permanente, usualmente a los 15 años de edad. Para la mandíbula severamente hipoplásica se recomienda un enfoque en varias etapas, reconociendo la dificultad de establecer un único protocolo de tratamiento para un grupo tan heterogéneo de pacientes con MHF, primando el injerto costocondral paralelo a la cirugía ortognática ${ }^{18}$.

El tratamiento convencional ortognático bimaxilar para nivelar el plano de oclusión canteado y la cirugía mandibular con o sin genioplastia pueden ser realizados según se requiera cuando se haya completado el crecimiento. Cuando la deficiencia del nervio facial amerita intervención, también se puede considerar la reactivación neurológica facial. Existe evidencia de que el uso de dispositivos de anclaje temporales en el maxilar podría reducir la necesidad de cirugía maxilar. Se requiere un seguimiento en el largo plazo para confirmar los hallazgos favorables iniciales. Como la MHF es una deformación en los tres sentidos del espacio, son útiles los modelos $3 \mathrm{D}$ para planificar los casos más complejos. El desarrollo y elección de injertos aloplásticos y/o autólogos va en aumento año a año. La reconstrucción del tejido blando y el contorneado facial han sido tradicionalmente llevados a cabo con colgajos libres de tejido blando. Los defectos más pequeńos adecuados para la realización de injertos de grasa de Coleman (autólogos) se han vuelto más populares. El tejido adiposo se obtiene de un sitio donante, injertado subcutáneamente en el área de la deficiencia. La técnica ha evolucionado con beneficios cada vez mejores en el largo plazo, y tiene la ventaja de ser mínimamente invasiva. Como procedimiento de camuflaje puede ser una alternativa razonable para un adolescente hasta que se le pueda ofrecer un tratamiento ortognático convencional. Aquellos pacientes con mutaciones genéticas conocidas, deben recibir asesoría de los médicos genetistas para sus planes futuros de paternidad ${ }^{2}$.

En el SG, el enfoque terapéutico no difiere en gran medida del de los pacientes con MHF. Como el espectro fenotípico es tan variable, el tratamiento debe tener un enfoque individual en todos los niveles y multidisciplinario. Comienza tempranamente, pero con el paso del 
tiempo se pueden ir observando nuevos signos en distintas partes del cuerpo y las anomalías diagnosticadas previamente se vuelven más severas debido principalmente al retraso del crecimiento ${ }^{6,7,19}$.

En los casos menos complejos, el tratamiento del síndrome varía según la edad y las asociaciones sistémicas, y es principalmente estético. En pacientes con hipoplasia mandibular, se pueden realizar reconstrucciones utilizando injertos costocondrales y una mandíbula poco desarrollada puede ser sometida a distracción ósea. Las correcciones quirúrgicas para la fisura labiopalatina pueden realizarse seguidas de corrección ortodóncica una vez que la mandíbula ha terminado su crecimiento. Las reconstrucciones quirúrgicas del oído externo pueden ser realizadas a los 6 a 8 ańos. En pacientes afectados más levemente, la reconstrucción mandibular puede realizarse en la adolescencia temprana; los dermoides epibulbares deben ser extirpados quirúrgicamente. Las anomalías estructurales de los ojos y orejas pueden ser corregidas con cirugía plástica. Los niños con este síndrome presentan un riesgo más elevado de alteraciones psicosociales, por lo tanto, debe existir una asesoría en este sentido tanto para el paciente como para su familia en cuanto a su integración a la comunidad y la toma de decisiones acerca de las cirugías y la adaptación a la asimetría facial. El pronóstico de esta enfermedad es bueno mientras sean casos poco complejos y sin asociaciones sistémicas ${ }^{14}$.

Los casos severos de SG pueden afectar muchos aspectos de la vida del paciente y a veces requieren intervención inmediata incluso desde el nacimiento. Por ejemplo, el paciente podría sufrir de SAHOS severo a causa de las anormalidades respiratorias. Los problemas mandibulares pueden desencadenar en alteraciones de dieta y malnutrición, y las anomalías palpebrales pueden guiar subsecuentemente a problemas de vista. Sin asistencia auditiva o reconstrucciones quirúrgicas, los individuos con microtia bilateral completa y atresia podrían desarrollar sordomudez permanente. Los ultrasonidos renales y cardiacos también han sido recomendados debido al riesgo aumentado de defectos de nacimiento en estas áreas ${ }^{14}$.

$\mathrm{Al}$ igual que en la MHF, existe controversia acerca del tiempo de intervención para la reparación quirúrgica de la deformidad mandibular en el SG. Cierta evidencia sugiere que el grado de deformidad permanece estable durante el crecimiento y que la asimetría mandibular no es progresiva. Contrariamente a dicha evidencia, otros sugieren que existe un canteo del plano oclusal y asimetría progresiva. Sin embargo, los tiempos quirúrgicos deben ser determinados individualmente, más allá del cuestionamiento de si la asimetría es o no más acrecentada con el tiempo. Si se observa una ventaja en ciertos pacientes sometidos a cirugías tempranamente (por ejemplo, cuando el resultado final será mejor con cirugía temprana), esto debe ser evaluado. Como con muchos procedimientos faciales reconstructivos, el tratamiento debería ser postergado hasta que exista madurez esqueletal. Sin embargo, en algunos casos la intervención quirúrgica temprana es ventajosa, como cuando existe una vía aérea comprometida, problemas psicosociales o cuando la cirugía temprana mejorará los resultados quirúrgicos finales. La reconstrucción orbitaria y malar utiliza un injerto de hueso de calota a través de una incisión coronal y exposición. Se recomienda que este procedimiento sea llevado a cabo después de los 6 años de edad ${ }^{20}$.

La reconstrucción maxilomandibular se aborda de mejor manera con cirugía ortognática tradicional a los 13 a 15 ańos de edad, al momento de la madurez esqueletal temprana. La reconstrucción de cóndilo y rama podría necesitar de injerto contocondral. A menudo las deformidades son suficientemente severas como para requerir una cirugía mandibular temprana con un injerto de costilla o bien distracción osteogénica. La intervención temprana debe realizarse en primera instancia con planificación y estudio de preparación para cirugía ortognática una vez terminado el crecimiento o la madurez esquelética ${ }^{20}$.

\section{Conclusiones}

La MHF y el SG son patologías heterogéneas, variables y de expresividad única en cada paciente, tanto en su etiología, como en su severidad y por ende en su tratamiento. Al ser alteraciones en espectro, afectan diversas estructuras del individuo de acuerdo a su severidad. Es importante evaluar y estudiar el caso como un trabajo interdisciplinario muy bien coordinado, ya que pueden presentar incluso alteraciones psicosociales y extracraneales, que deben ser exploradas y tratadas a tiempo. El inicio del tratamiento temprano va a permitir que el manejo clínico en las anomalías leves sea controlado basándose en la planificación del tratamiento y en los casos severos coordinar la terapia ortodóncica quirúrgica en el mejor tiempo posible.

\section{Referencias bibliográficas}

1. Shrestha U, Adhikari S. Craniofacial Microsomia: Goldenhar Syndrome in Association with Bilateral Congenital Cataract. Case Rep Ophthalmol Med. 2015;2015:13. DOI: $10.1155 / 2015 / 435967$.

2. Akram A, McKnight M, Bellardie H, Beale V, Evans R. Craniofacial malformations and the orthodontist. Br Dent J. 2015;218(3):129-141. DOI: 10.1038/ sj.bdj.2015.48.

3. Tuin J, Tahiri Y, Paliga J, Taylor J, Bartlett S. Distinguishing Goldenhar Syndrome from Craniofacial Microsomia. J Craniofac Surg. 2015;26(6):1887-1892. DOI: 10.1097/SCS.0000000000002017.

4. Birgfeld C, Heike C. Craniofacial Microsomia. Semin Plast Surg. 2012;26(02):091-104. DOI: $10.1055 / \mathrm{s}-$ 0032-1320067.

5. Cohen N, Cohen E, Gaiero A, Zecca S, Fichera G, Baldi F, et al. Maxillofacial features and systemic malformations in expanded spectrum Hemifacial Microsomia. Am J Med Gene. Part A. 2017;173(5):1208-1218. DOI: 10.1002/ajmg.a.38151. 
6. Bogusiak K, Puch A, Arkuszewski P. Goldenhar syndrome: current perspectives. World J Pediatr. 2017; 13(5): 405 - 415. DOI: 10.1007/s12519-017-0048-z.

7. Pati A, Bhuyan R, Bhuyan S, Nayak B. Goldenhar syndrome: A rare case report. J Oral Maxillofac Pathol. 2016;20(2):328. DOI: 10.4103/0973-029X.185907.

8. Brandstetter K, Patel K. Craniofacial Microsomia. Facial Plast Surg Clin North Am. 2016;24(4):495-515. DOI: 10.1016/j.fsc.2016.06.006.

9. Wolford L, Perez D. Surgical Management of Congenital Deformities with Temporomandibular Joint Malformation. Oral Maxillofac Surg Clin North Am. 2015;27(1):137-154. DOI: $10.1016 / j$. coms.2014.09.010.

10. Leiva N, Castellón L, Agurto P, Morovic C. Microsomía Hemifacial. Tratamiento de un paciente en crecimiento. Rev Dent Chile 2010;101(3):31-7.

11. Véliz S, Agurto P, Leiva N. Microsomíahemifacial. Revisión de la literatura. Rev Fac Odontol Univ Antioq. 2016; 27(2): 404-424. DOI: 10.17533/udea.rfo. v27n2a9.

12. Birgfeld C, Heike C. Craniofacial Microsomia. Semin Plast Surg. 2012;26(02):91-104. DOI: $10.1055 / \mathrm{s}$ 0032-1320067.

13. Tuin A, Tahiri Y, Paine K, Paliga J, Taylor J, Bartlett S. Clarifying the Relationships among the Different Features of the OMENS+ Classification in Craniofacial Microsomia. Plast Reconstr Surg. 2015;135(1):149e-156e. DOI: $10.1097 /$ PRS.0000000000000843.
14. Seethalakshmi C. Goldenhar Syndrome - Review with Case Series. J Clin Diagn Res. 2014;(4):ZD17-ZD19. DOI: $10.7860 / J C D R / 2014 / 7926.4260$.

15. Caron C, Pluijmers B, Joosten K, Mathijssen I, van der Schroeff M, Dunaway D, et al. Obstructive sleep apnoea in craniofacial microsomia: a systematic review. Int J Oral Maxillofac Surg. 2015;44(5):592-598. DOI: 10.1016/j.ijom.2015.01.023.

16. Weichman K, Jacobs J, Patel P, Szpalski C, Shetye P, Grayson B, et al. Early Distraction for Mild to Moderate Unilateral Craniofacial Microsomia. Plast Reconstr Surg. 2017;139(4):941e-953e. DOI: 10.1097/ PRS.0000000000003223.

17. Ascenço A, Balbinot P, Junior I, D'Oro U, Busato L, da Silva Freitas R. Mandibular Distraction in Hemifacial MicrosomiaIs Not a Permanent Treatment. J Craniofac Surg. 2014;25(2):352-354. DOI: 10.1097/01. scs.0000436741.90536.bf.

18. Pluijmers B, Caron C, Dunaway D, Wolvius E, Koudstaal M. Mandibular reconstruction in the growing patient with unilateral craniofacial microsomia: a systematic review. Int J Oral Maxillofac Surg. 2014;43(3):286-295. DOI: $10.1016 /$ j.ijom.2013.11.001.

19. Bogusiak K, Arkuszewski P, Skorek-Stachnik K, Kozakiewicz M. Treatment Strategy in Goldenhar Syndrome. J Craniofac Surg. 2014;25(1):177-183. DOI: $10.1097 /$ SCS.0000000000000387.

20. Alfi D, Lam D, Gateno J. Branchial Arch Syndromes. Atlas Oral Maxillofac Surg Clin North Am. 2014;22(2):167173. DOI: $10.1016 /$ j.cxom.2014.04.003. 